\title{
THE SOLUTION OF THE FUNCTIONAL EQUATION OF D'ALEMBERT'S TYPE FOR COMMUTATIVE GROUPS
}

\author{
A.L. RUKHIN \\ Department of Statistics, Purdue University \\ West Lafayette, Indiana 47907 U.S.A. \\ (Received June 2, 1980 and in revised form December 11, 1980)
}

ABSTRACT. A functional equation of the form $\phi_{1}(x+y)+\phi_{2}(x-y)=\sum_{i}^{p} \alpha_{i}(x) \beta_{i}(y)$, where functions $\phi_{1}, \phi_{2}, \alpha_{i}, \beta_{i}, i=1, \ldots, n$ are defined on a coimutative group, is solved. We also obtain conditions for the solutions of this equation to be matrix elements of a finite dimensional representation of the group.

KEY WORDS AND PHRASES. D'Alembert's (cosinel functional equation, finite dimensional representations, commutative groups, fields of characteristic zero.

1980 MATHEMATICS SUBJECT CLASSIFICATION CODES. Primary 39B50, Secondary 20K99, 43499

1. INTRODUCTION.

Consider the functional equation

$$
\phi_{1}(x+y)+\phi_{2}(x-y)=\alpha_{1}(x) \beta_{1}(y)+\ldots+\alpha_{n}(x) \beta_{n}(y),
$$

where $\phi_{1}, \phi_{2}, \alpha_{i}, \beta_{i}, i=1, \ldots, n$ are functions given on a commutative group $C_{d}$, taking values in a field $\mathrm{F}$ of characteristic zero.

$$
\begin{aligned}
\text { Clearly, if } f(x) & =\phi_{1}(x)-\phi_{2}(x), g(x)=\phi_{1}(x)+\phi_{2}(x) \text {, then } \\
f(x+y)-f(x-y) & =\sum_{i=1}^{n} \alpha_{i}(x)\left[\beta_{i}(y)-\beta_{i}(-y)\right]=\sum_{j=1}^{m} h_{j}(x) k_{j}(y)
\end{aligned}
$$

and

$$
g(x+y)+g(x-y)=\sum_{i=1}^{n} \alpha_{i}(x)\left[\beta_{i}(y)+\beta_{i}(-y)\right]=\sum_{i=1}^{p} u_{i}(x) v_{i}(y),
$$


where the functions $h_{j}, k_{j}, j=1, \ldots, m$ and $u_{i}, v_{i}, i=1, \ldots, p$ are linearly independent. Therefore it suffices to consider the case when $\phi_{1}=\phi_{2}$ or $\phi_{1}=-\phi_{2}$ in (1.1). Note that linear independence of $h_{j}$ and $u_{i}$ implies $k_{j}(-y)=-k_{j}(y), j=1$, $\ldots, m$ and $v_{i}(-y)=v_{i}(y), i=1, \ldots, p$.

The equation (1.1) can be viewed as a generalization of D'Alembert's (cosine) functional equation

$$
\phi(x+y)+\phi(x-y)=2 \phi(x) \phi(y),
$$

which has been much studied (cf Acze1 [1, p. 176], Corovei [3], Hosszu [6], Kannappan [7], O'Connor [12], Rejto [14]). It a1so arises in statistical applications (Rukhin [15]).

The functional equations (1.2) and (1.3) follow from the equation

$$
\phi(x+y)=\alpha_{1}(x) \beta_{1}(y)+\ldots+\alpha_{m}(x) \beta_{m}(x),
$$

which also was an object of detailed study. Clearly (1.5) implies that the space obtained by taking finite linear combinations of translates of $\phi$ is of finite dimension, and this of course means that $\phi$ is a matrix element of a finite dimensional representation of the group $c_{8}$. It is known (cf Engert [4], Laird [8], Stone [17]) that, for a locally compact group, every finite dimensional (or only closed in the space of all continuous functions) translation invariant subspace consists of exponential polynomials. In other terms must have the form

$$
\phi(x)=\sum_{i=1}^{n} p_{i}(x) g_{i}(x),
$$

where $g_{i}$ are multiplicative homomorphisms of $\mathcal{G}$ into $\mathfrak{F}$ and $p_{i}$ are polynomials in different additive homomorphisms of $\mathcal{G}$ into $\mathfrak{F}$. Actually, the solutions of the functional equation (1.5) are known to be of such a form in a more general situation when $\mathcal{G}$ is a groupoid or a semigroup and $\mathfrak{F}$ is a commutative ring (see Aczel [2], McKiernan [10], [11]). However, as we shall see, not every exponential polynomial is a solution of (1.5) in the nonlocally compact case.

In Section 3 we obtain the general form of the solutions $f$ and $g$ of equations (1.2) and (1.3). These solutions are expressed as linear combinations of matrix elements of inequivalent finite dimensional representations of the group $\mathcal{C}$ 
and also of terms involving homomorphisms of $\hat{\zeta}$ into a vector space $\mathfrak{f}^{\mathrm{n}}$ over the field $\xi$ and homomorphisms of $\mathcal{G}$ into additive matrix group over $\exists$. Section 2 contains some preliminary results about polynomials on Abelian groups. The discussion of the main result is given in Section 4, where the equations (1.2) and (1.3) are proved to have all solutions being exponential polynomials. We also prove that while every solution of (1.2) is a solution of (1.5), there are solutions of (1.3) which are not matrix elements of any finite dimensional representation of $\mathcal{G}$, i.e. which do not satisfy (1.5), and give sufficient conditions for a solution of (1.3) to have such form.

\section{POLYNOMIALS OVER COMMUTATIVE GROUPS.}

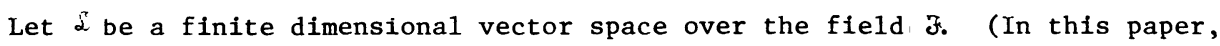
$\mathcal{L}$ will be a vector space $\mathcal{J}_{n}$ of all $\mathrm{n} \times \mathrm{n}$ matrices over the field $\mathcal{F}$, or the vector space $\xi^{\text {n }}$ of dimension $\mathrm{n}$ on $\xi^{3}$. If $\psi$ is an $\mathcal{L}$-valued function defined on the Abelian group $g$, then $L(x), x \in G$ is the translation operator, $L(x)_{\psi}(\cdot)=\psi(\cdot+x)$. Thus $L$ is a regular representation of $\mathcal{G}$ which acts in the linear space spanned by the translates of the function $\psi$. The function $\psi$ is called a polynomial if, for some $n,(L(x)-I)^{n+1} \psi(y) \equiv 0$ for all $x, y \in C_{g}$. The smallest number $n$ for which this identity holds is called the degree of the polynomial.

Thus a polynomial of degree one satisfies the identity

$$
\psi(\mathrm{x}+\mathrm{y})+\psi(\mathrm{x}-\mathrm{y})=2 \psi(\mathrm{x}) .
$$

If $\quad 2 f=f$ this condition implies that $\psi(x)=x(x)+c$, where $c \in \mathcal{L}$, $x \in \operatorname{Hom}(\zeta, \mathcal{L})$; i.e., $x(x+y)=x(x)+\chi(y)$ for all $x, y \in \mathcal{C}$.

A polynomial $\psi$ is said to be homogenous, if

$$
(\mathrm{L}(\mathrm{x})-\mathrm{I}) \mathrm{n}_{\psi(\cdot)}=\mathrm{n} ! \psi(\mathrm{x}) .
$$

The following elementary results [9] will be used in Section 3 .

10. If $\varphi$ is a homogenous polynomial of degree $n$, then for all integer $j$

$$
\varphi(j x)=j^{n} \varphi(x), x \in C_{i} .
$$

20 . If $\psi$ is a polynomial of degree $n$, then $\psi(x)=(L(x)-I)_{\psi}^{n}(y)$ does not dedepend on $y$ and is an homogenous polynomial of degree $n$ in $x$.

$3^{\circ}$. If $\psi$ is a polynomial of degree $n$, then $\left(L\left(x_{1}\right)-I\right) \ldots\left(L\left(x_{j}\right)-I\right) \psi(x)$ is a poly- 
nomial in $x$ of degree $n-j$.

$4^{\circ}$. If $\psi$ is a polynomial of degree $n$, then

$$
\psi(\mathrm{x})=\varphi_{\mathrm{n}}(\mathrm{x})+\ldots+\varphi_{0}(\mathrm{x}),
$$

where $\Psi_{j}(x)$ is a homogenous polynomial of degree $j, j=0, \ldots, n$. One has

$$
\Psi_{n}(x)=\frac{1}{n !}(L(x)-I)^{n} \psi(\cdot)
$$

and for $j=n-1, \ldots, 0$,

$$
\varphi_{j}(x)=\frac{1}{j !}(L(x)-I)^{j}\left(f(\cdot)-\varphi_{n}(\cdot)-\ldots-\varphi_{j+1}(\cdot)\right) .
$$

$5^{\circ}$. If $\psi$ is a homogenous polynomial of degree $n$, then $\varphi(x)=\chi(x, \ldots, x)$ where $x\left(x_{1}, \ldots, x_{n}\right)$ is a symmetric function of $x_{1}, \ldots, x_{n}$ and for fixed $x_{2}, \ldots, x_{n}, x\left(\cdot, x_{2}, \ldots, x_{n}\right) \in \operatorname{Hom}(\mathcal{\zeta}, \mathfrak{L})$.

If $\varphi$ is a polynomial of even degree and $2 j=j \quad$ then in all formulas above $\mathrm{L}(\mathrm{x})-\mathrm{I}$ can be replaced by $\mathrm{L}(\mathrm{x} / 2)-\mathrm{L}(-\mathrm{x} / 2)$.

If $k \in \mathfrak{F}^{n}$ and $k \in \mathfrak{f}^{\star n}$, where $\mathfrak{F}^{\star n}$ is the dual space, then $\langle\mathrm{h}, \mathrm{k}\rangle$ will always denote the value of the linear functional $k$ on the element $h$. With this convention, equation (1.2), for instance, can be rewritten as

$$
f(x+y)-f(x-y)=\langle h(x), k(y)\rangle,
$$

where $h(x), \in \mathfrak{F}^{\mathrm{m}}$, and $k(y) \in \mathfrak{F}^{\mathrm{km}}$. Also, $A^{t}$ will denote the transpose of a linear transformation A.

3. THE MAIN RESULT.

A structure theorem for the solutions of the functional equations (1.2) and (1.3) is obtained in this Section.

Theorem 1. Assume that $\mathcal{C}$ is a commutative group such that $2 \mathfrak{f}=\mathfrak{f}$. function $f$ taking values in an algebraically closed field $\mathfrak{F}$ of characteristic zero is a solution of the equation (1.2) with linearly independent functions $h_{j}, k_{j}, j=1, \ldots, m$ if, and only if, there exist nonnegative integers $\mathrm{m}_{1}, \ldots, \mathrm{m}_{\mathrm{R}}, \mathrm{m}_{1}+\ldots+\mathrm{m}_{\mathrm{R}}=\mathrm{m}$ such that

$$
\begin{aligned}
f(x) & =\left\langle S(x) f_{1}, \varphi(x)\right\rangle+\left\langle T(x) Q_{1} \varphi(x)\right\rangle \\
& +\sum_{r=2}^{R}\left[\left\langle F_{r}(x) f_{r}, l_{r}\right\rangle+\left\langle F_{r}(-x) d_{r}, l_{r}\right\rangle\right]+c .
\end{aligned}
$$

Here $q \in \operatorname{Hom}\left(c_{i}, \mho^{*} \mathrm{~m}_{1}\right), S(x)=\sum_{k=0}^{m_{1}-1} H^{k}(x, x) /(2 k+1) !, T(x)=\sum_{k=0}^{m_{1}-1} H^{k}(x, x) /(2 k+2) !$, where 
for each $y \in \mathcal{C}_{H}(\cdot, y) \in \operatorname{Hom}\left(\mathcal{C}_{\partial \mathfrak{F}_{\mathrm{m}_{1}}}\right), \mathrm{H}(\mathrm{x}, \mathrm{y})=\mathrm{H}(\mathrm{y}, \mathrm{x}), \mathrm{H}^{\mathrm{m}}{ }^{1}(\mathrm{x}, \mathrm{y})=0$ if $\mathrm{m}_{1} \geq 1, \mathrm{H}^{2}(\mathrm{x}, \mathrm{y})=\mathrm{H}(\mathrm{x}, \mathrm{x}) \mathrm{H}(\mathrm{y}, \mathrm{y}), \mathrm{H}^{\mathrm{t}}(\mathrm{x}, \mathrm{x}) \varphi(\mathrm{y})=\mathrm{H}^{\mathrm{t}}(\mathrm{x}, \mathrm{y}) \varphi(\mathrm{x})$ for a11 $\mathrm{x}, \mathrm{y}$; $\mathrm{F}_{\mathrm{r}}, \mathrm{r}=2, \ldots, \mathrm{R}$ are pairwise inequivalent matrix representations of the group $\mathrm{G}$ of degree $m_{r}$ where all eigenvalues of $F_{r}$ are equal and not identically one; $Q_{r}$ are invertible linear operators from $\mathfrak{f}^{{ }^{*} \mathrm{~m}_{\mathrm{r}}}$ to $\mathfrak{f}^{\mathrm{m}_{\mathrm{r}}}$, $H(x, x) Q_{1}=Q_{1} H^{t}(x, x), F_{r}(x) Q_{r}=Q_{r} F_{r}^{t}(x), r=2, \ldots, R ; f_{1} \in \mathfrak{F}^{m_{1} \ell_{r}} \in \mathfrak{F}^{*} m_{r}$ $f r, d \underset{r}{\in} \mathfrak{F}^{m_{r}}, r=2, \ldots, R, f_{r}+d_{r}=2 Q_{r} \ell_{r}, r=2, \ldots, R, c \in \mathfrak{F}$. Also the vectors $C(x) f_{1}+s(x) Q_{1} \varphi(x), x \in \mathcal{G}$ span $\mathfrak{F}^{m_{1}}$ and the vectors $s^{t}(x) \varphi(x)$ span $\mathcal{F}^{*} \mathrm{~m}_{1}, \mathrm{C}(\mathrm{x})=\sum_{\mathrm{k}=0}^{\mathrm{m}_{1}-1} \mathrm{H}^{\mathrm{k}}(\mathrm{x}, \mathrm{x}) /(2 \mathrm{k}) !$; the spaces $\mathcal{F}^{\star} \mathrm{m}_{\mathrm{r}}$ and $\mathcal{F}^{\mathrm{m}_{\mathrm{r}}}, \mathrm{r}=2, \ldots, \mathrm{R}$ are spanned by the vectors $\left[F_{r}^{t}(x)-F_{r}^{t}(-x)\right] \ell_{r}, x \in \mathcal{C}$ and by the vectors $F_{r}(x) f_{r}-F{ }_{r}(-x) d_{r} x \in \mathcal{C}_{\text {, }}$, corresponding1y. The representation (3.1) is unique up to equivalence for matrices $H(x, x)$ and $\mathrm{F}_{\mathrm{r}}(\mathrm{x}), \mathrm{r}=2, \ldots, \mathrm{R}$.

We do not prove the next Theorem 2 since its proof is analogous to that of Theorem 1 .

Theorem 2. Under assumptions of Theorem 1 a function $g$ is a solution of the equation (1.3) with linearly independent function $u_{i}, v_{i} i=1, \ldots, p$ if, and only if, there exist nonnegative integers $\mathrm{p}_{1}, \ldots, \mathrm{p}_{\mathrm{R}}, \mathrm{p}_{1}+\ldots+\mathrm{p}_{\mathrm{R}}=\mathrm{p}$, such that

$$
\begin{aligned}
g(x)= & <C(x) Q_{1} g_{1}, a_{1}>+<S(x) \psi(x), a_{1}> \\
& +\sum_{r=2}^{R}\left[\left\langleF_{r}(x) g_{r}, a_{r}>+\left\langle F_{r}(-x) b_{r}, a_{r}>\right]+c .\right.\right.
\end{aligned}
$$

Here $C(x), S(x), F_{r}(x), Q_{r}$ and $Q_{r}$ have the same meaning as in Theorem 1 with $m_{r}$ replaced by $p_{r}, \psi \in \operatorname{Hom}\left(C_{d} \mathfrak{J}^{p^{1}}\right), b_{r}, g_{r} \in \mathfrak{F}^{m_{r}, g_{r}-b}{ }_{r}=2 Q_{r} a_{r}, a_{r} \in \mathfrak{F}^{\star}{ }^{m_{r}}, r=2, \ldots, R, c \in \mathfrak{F}$. The vectors $C^{t}(x) a_{1}, x \in C_{\mathcal{*}}$ span $\mathfrak{F}^{*} \mathrm{p}_{1}$, and the vectors $C(x) a_{1}+S(x) \psi(x), x \in C_{d}$, span $\mathcal{F}^{\mathrm{P}_{1}}$; the spaces $\mathcal{F}^{\mathrm{P}_{\mathrm{r}}}$ and $\mathcal{F}^{*} \mathrm{P}_{\mathrm{r}} \mathrm{f}=2, \ldots, \mathrm{R}$ are spanned by the vectors $\mathrm{F}_{\mathrm{r}}(\mathrm{x}) \mathrm{g}_{\mathrm{r}}+\mathrm{F}_{\mathrm{r}}(-\mathrm{x}) \mathrm{b}_{\mathrm{r}}$ and by the vectors, $\left[\mathrm{F}_{\mathrm{r}}^{\mathrm{t}}(\mathrm{x})+\mathrm{F}_{\mathrm{r}}^{\mathrm{t}}(-\mathrm{x})\right] \mathrm{a}_{\mathrm{r}}$ correspondingly. The matrix functions $H(x, x)$ and $F_{r}(x) r=2, \ldots, R$ are defined uniquely up to equivalence. We break up the proof of Theorem 1 into three lemmas.

Lemma 1. Assume that the functional equation

$$
f_{1}(x+y)-f_{1}(x-y)=\langle w(x), k(y)\rangle
$$


has a symmetric solution $f_{1}, f_{1}(-x)=f_{1}(x)$. Then the vector function $w$ has the form, $w(x)=B k(x)$, where $B$ is an invertible linear operator from $\mathfrak{F}^{*} \mathrm{~m}$ to $\mathfrak{F}^{\mathrm{m}}$, $B^{t}=B$. Also $k(x)=T^{t} \ell(x)$, where $T$ is an invertible linear operator from $\mathfrak{F}^{\star} m$ to $\mathfrak{J}^{\star} \mathrm{m}$ and there exist nonnegative integers $\mathrm{m}_{1}, \ldots, \mathrm{m}_{\mathrm{R}}$ such that $\mathfrak{F}^{\star} \mathrm{m}=\mathfrak{F}^{\star} \mathrm{m}_{1} \oplus \ldots \oplus \mathfrak{F}^{\mathrm{m}_{\mathrm{r}}}$ and the projections $\ell_{r}$ of $\ell$ onto $\mathfrak{E}^{\star} \mathrm{m}_{\mathrm{r}}$ satisfy the following functional equation,

$$
\ell_{r}(x+y)+\ell_{r}(x-y)=2 B_{r}(y) \ell_{r}(x) .
$$

Here $B_{r}(y)$ is an upper triangular matrix of dimension $m_{r}$ with the same diagonal elements $b_{(r)}(y), b^{(r)}(y) \neq b^{(s)}(y), r \neq s$, such that $Q_{r} B_{r}(y)=B_{r}^{t}(y) Q_{r}$ with some invertible operators $Q_{r}, Q_{r}^{t}=Q_{r}, r=1, \ldots, R$, and

$$
B_{r}(x+y)+B_{r}(x-y)=2 B_{r}(x) B_{r}(y) \text {. }
$$

Proof of Lemma 1 . Since $\mathrm{f}_{1}$ is symmetric

$$
\langle w(x), k(y)\rangle=\langle w(y), k(x)\rangle \text {. }
$$

Therefore there exists an invertible linear operator B from $\mathfrak{f}^{\star} \mathrm{m}$ to $\mathfrak{f}^{\mathrm{m}}$ such that $B^{t}=B$ and for all $x \in C$

$$
w(x)=B k(x) .
$$

Now let $\mathrm{V}$ denote the linear space over $\mathcal{F}$ spanned by the translates $\mathrm{f}(\cdot+\mathrm{x})$, $x \in C_{\delta}$ of the function $f$ which satisfies (1.2). Then the regular representation $\mathrm{L}(\mathrm{x}): \mathrm{L}(\mathrm{x}) \mathrm{g}=\mathrm{g}(\cdot+\mathrm{x}), \mathrm{g} \in \mathrm{V}$ acts in $\mathrm{V}$, and the functional equation (1.2) means that

$$
[L(y)-L(-y)] f=\sum_{j=1}^{m} k_{j}(y) h_{j} \text {. }
$$

Here $f$ denotes the (cyclic) vector of $V$ corresponding to the function $f(\cdot)$, and $h_{1}, \ldots, h_{m}$ are vectors from $v$ which correspond to the functions $h_{1}(\cdot), \ldots, h_{m}(\cdot)$. If $V_{\text {_ }}$ denotes the subspace of $V$ spanned by the vectors $[L(y)-L(-y)] f, y \in C_{d}$, then it follows from (3.3) that $\mathrm{V}_{\text {- }}$ has dimension $\mathrm{m}$. Also, as is easy to see, $\mathrm{V}_{-}$is invariant under a11 operators $L(x)+L(-x), x \in G$.

Let $A(x)$ denote the restriction of the operator $[L(x)+L(-x)] / 2$ on $V_{-}$. Then,

$$
2 \sum_{j=1}^{m} k_{j}(y) A(x) h_{j}=\sum_{j=1}^{m}\left[k_{j}(x+y)+k_{j}(-x+y)\right] h_{j},
$$


so that

$$
k(x+y)+k(-x+y)=2 A^{t}(x) k(y)
$$

It is evident that all matrices $A(x)$ commute. Therefore (see Suprunenko and Tyshkevich [18] p. 16) the whole space $\mathfrak{F}^{\star m}$ can be represented as a direct sum of invariant subspaces $W_{r}$, with respect to all $A(x)$, for $r=1, \ldots, R$. The irreducible parts of $A(x) \mid W_{r}$ are equivalent, while for $r \neq s$ the irreducible parts of $A(x) \mid W_{r}$ and $A(x) \mid W_{s}$ are not equivalent. Since the field $\mathfrak{F}$ is algebraically closed, Shur's lemma shows that all irreducible parts of $A(x) \mid W_{r}, r=1, \ldots, R$, are one-dimensional operators. Thus all matrices $A(t)$ have the form $A(x)=T^{-1} B^{t}(x) T$, where $B(x)$ is a quasi-diagonal matrix with blocks $B_{1}(x), \ldots, B_{R}(x)$ on the principal, diagonal, and $\mathrm{B}_{\mathrm{r}}(\mathrm{x})$ is an upper triangular matrix of dimension $\mathrm{m}_{\mathrm{r}}=\mathrm{dim} \mathrm{w}_{\mathrm{r}}, \mathrm{r}=1, \ldots, \mathrm{R}$ with the same diagonal elements $b^{(r)}(x), b^{(r)}(x) \neq b^{(s)}(x), \quad r \neq s . \quad$ Clearly $m=m_{1}+\ldots+m_{R}$ and all matrices $B_{r}(x), r=1, \ldots, R$ commute.

Let $Q=T_{B T} T^{t}$ Then $Q^{t}=Q, Q$ is invertible and $Q B(x)=B^{t}(x) Q$. Because of Shur's lemma $Q=Q_{1} \oplus \ldots \oplus Q_{r}$ where $Q_{r}$ is of dimension $m_{r}$, and $Q_{r} B_{r}(x)=B_{r}^{t}(x) Q_{r}, r=1, \ldots, R$. Also, if $k(y)=T^{t} \ell(y)$ then

$$
\ell(\mathrm{x}+\mathrm{y})+\ell(\mathrm{x}-\mathrm{y})=2 \mathrm{~B}(\mathrm{y}) \ell(\mathrm{x}) .
$$

Let $\ell(y)=\ell_{1}(y) \oplus \ldots \theta l_{R}(y)$ with $\ell_{r} \in \mathcal{F}^{\star} m_{r}, r=1, \ldots, R$ be the partition of $\ell(y)$ into direct sum corresponding to that of the matrix $B(x)$. Then for $r=1, \ldots, R$

$$
\ell_{r}(x+y)+\ell_{r}(x-y)=2 B_{r}(y) l_{r}(x) .
$$

It is easy to deduce from the definition of $A(x)$ that the matrices $A(x)$ satisfy D'Alembert's functional equation

$$
A(x+y)+A(x-y)=2 A(x) A(y) .
$$

It follows from (3.6) that

$$
B_{r}(x+y)+B_{r}(x-y)=2 B_{r}(x) B_{r}(y), \quad r=1, \ldots, R,
$$

so that Lemma 1 is proven.

Lemma 2. For $r=1, \ldots, R$

$$
b^{(r)}(x)=\left[x_{r}(x)+x_{r}(-x)\right] / 2,
$$

where $x_{r}$ is a multiplicative homomorphism of $\zeta_{\text {into }} \mathcal{F}, \chi_{r}(x+y)=\chi_{r}(x) x_{r}(y)$. If $\chi_{\mathbf{r}}$ is not identically one, then 


$$
\ell_{r}(x)=\left[G_{r}(x)-G_{r}(-x)\right] \ell_{r},
$$

where $G_{r}(x)$ are lower triangular matrices of dimension $m_{r}$ with all diagonal elements equal to $X_{r}(x), G_{r}(x+y)=G_{r}(x) G_{r}(y) ; Q_{r} G_{r}(x)=G_{r}^{t}(x) Q_{r}$ with invertible $Q_{r}, Q_{r}^{t}=Q_{r}, \ell_{r} \in \mathfrak{F}^{\star} \mathrm{m}$.

Proof of Lemma 2. It follows from (3.7) that

$$
b^{(r)}(x+y)+b^{(r)}(x-y)=2 b^{(r)}(x) b^{(r)}(y) .
$$

A11 solutions of this D'Alembert's functional equation are known to be of the form (cf. Kannappan [7])

$$
b^{(r)}(x)=\left[x_{r}(x)+x_{r}(-x)\right] / 2,
$$

where $\chi_{r}$ is a multiplicative homomorphism of $C_{f}$ into :

$$
x_{r}(x+y)=x_{r}(x) x_{r}(y) \text {. }
$$

If $x_{r}$ is not identically one there exists $x_{0} \in C_{f}$ such that $\chi_{r}\left(2 x_{0}\right) \neq 1$ and the matrix $B_{r}^{2}\left(x_{0}\right)-I=\left[B_{r}\left(2 x_{0}\right)-I\right] / 2$ is nonsingular. Moreover, one can find a nonsingular lower triangular matrix $G_{r}$ such that $G_{r}^{2}=B_{r}^{2}\left(x_{0}\right)-I$. Indeed,

$$
B_{r}^{2}\left(x_{0}\right)-I=\left[\left(x_{r}\left(x_{0}\right)-x_{r}\left(-x_{0}\right)\right) / 2\right]^{2}\left[I+P_{r}\right],
$$

where $\mathrm{P}_{\mathrm{r}}$ is a nilpotent matrix, $\mathrm{P}_{\mathrm{r}}^{\mathrm{m}_{\mathrm{r}}}=0$.

Thus one can put

$$
G_{r}=\left[\left(x_{r}\left(x_{0}\right)-x_{r}\left(-x_{0}\right)\right) / 2\right]\left[I+P_{r} / 2+\sum_{i=2}^{m_{r}^{-1}} \frac{(-1)^{i+1}(2 i-1) ! !}{2^{i} i !} P_{r}^{i}\right] \text {. }
$$

Clearly $G_{r}$ commutes with all matrices $B_{r}(x)$ and $Q_{r} G_{r}=G_{r}^{t} Q_{r}$.

Now let

$$
\begin{aligned}
G_{r}(x) & =G_{r}^{-1}\left[B_{r}(x)\left(G_{r}-B_{r}\left(x_{0}\right)\right)+{ }_{r}\left(x+x_{0}\right)\right] \\
& =B_{r}(x)-G_{r}^{-1}\left[B_{r}(x) B_{r}\left(x_{0}\right)-B_{r}\left(x+x_{0}\right)\right] .
\end{aligned}
$$

It is easy to check (cf. [5]) that

$$
G_{r}(x+y)=G_{r}(x) G_{r}(y),
$$

and

$$
Q_{r} G_{r}(x)=G_{r}^{t}(x) Q_{r} \text {. }
$$

Evidently $G_{r}(x)$ and $G_{S}(x)$ are inequivalent for $r \neq s$ and 
$G_{r}(x)+G_{r}(-x)=2 B_{r}(x)-G_{r}^{-1}\left[2 B_{r}(x) B_{r}\left(x_{0}\right)-B_{r}\left(x+x_{0}\right)-B_{r}\left(-x+x_{0}\right)\right]=2 B_{r}(x)$.

It is also clear that $G_{r}(x)$ is a lower triangular matrix with all diagonal elements (and hence eigenvalues) equal to $x_{r}(x)$.

It follows from (3.5)

$$
l_{r}(x+y)+l_{r}(y-x)=2 B_{r}(x) l_{r}(y),
$$

so that

$$
\ell_{r}(x-y)=B_{r}(y) l_{r}(x)-B_{r}(x) l_{r}(y) .
$$

Using again (3.5) we see that

$$
\begin{aligned}
2 B_{r}(x)\left[l_{r}(x+y)+l_{r}(x-y)\right] & =2 B_{r}(y) l_{r}(2 x) \\
& =2 B_{r}(y)\left[B_{r}(x-y) l_{r}(x+y)+B_{r}(x+y) l_{r}(x-y)\right] .
\end{aligned}
$$

Now one deduces from (3.7)

$$
B_{r}(y) B_{r}(x-y)=\left[B_{r}(x)+B_{r}(x-2 y)\right] / 2
$$

and

$$
B_{r}(y) B_{r}(x+y)=\left[B_{r}(x)+B_{r}(x+2 y)\right] / 2 .
$$

Thus

$$
\left[B_{r}(x)-B_{r}(x-2 y)\right] \ell_{r}(x+y)=-\left[B_{r}(x)-B_{r}(x+2 y)\right] \ell_{r}(x-y) .
$$

It is easy to check that

$$
B_{r}(x)-B_{r}(x-2 y)=\left[G_{r}(y)-G_{r}(-y)\right]\left[G_{r}(x-y)-G_{r}(-x+y)\right] / 2,
$$

and

$$
-B_{r}(x)+B_{r}(x+2 y)=\left[G_{r}(y)-G_{r}(-y)\right]\left[G_{r}(x+y)-G_{r}(-x-y)\right] / 2 .
$$

Let $K_{r}=\left\{x: x_{r}(2 x)=1\right\}$. If $x \notin k_{r}$ the matrix $G_{r}(x)-G_{r}(-x)$ is nonsingular.

Thus if $y \notin K_{r}\left[G_{r}(x+y)-G_{r}(-x-y)\right] d_{r}(x-y)=\left[G_{r}(x-y)-G_{r}(-x+y)\right] e_{r}(x+y)$. It follows that the relations $\mathrm{x}+\mathrm{y} \notin \mathrm{K}_{\mathrm{r}}$ and $\mathrm{x}-\mathrm{y} \notin \mathrm{K}_{\mathrm{r}}$ imply

$$
\left[G_{r}(x+y)-G_{r}(-x-y)\right]^{-1} \ell_{r}(x+y)=\left[G_{r}(x-y)-G_{r}(-x+y)\right]^{-1} \ell_{r}(x-y) .
$$

In other words for $a \notin \mathrm{K}_{\mathrm{r}}$

$$
\ell_{r}(z)=\left[G_{r}(z)-G_{r}(-z)\right] \ell_{r}
$$

with some vector $\ell_{r}$ if $z$ has the form $z=x+y$ with $y \notin K_{r}$ and $x-y \notin K_{r}$ or $z=x+2 y, x, y \notin k_{r}$. We prove now that every element $z \notin K_{r}$ has this form.

If there exists $x_{0} \in K_{r}$ such that $x_{r}\left(x_{0}\right)+1$ we put $z=\left(z+x_{0}\right)-x_{0}$. 
Clearly $z+x_{0} \notin K_{r}$ and $x_{0} / 2 \notin K_{r}$. If for all $x \in K_{r}$ one has $x_{r}(x)=1$, then we show that $z=x+y$ with $x, y \notin K_{r}$. Indeed in this case it suffices to take $=\mathrm{y}=\mathrm{z} / 2$.

Thus (3.8) holds for all $\mathrm{z}_{\mathrm{r}} \notin \mathrm{K}_{\mathrm{r}}$. We prove now that (3.8) is valid for all $z_{1} \in C_{0}$. Let $z \in K_{r}, x \notin K_{r}$, then $x+z_{1} \notin K_{r}$ and $x-z \notin K_{r}$. Therefore

$$
\begin{aligned}
\ell_{r}(z+x)+\ell_{r}(z-x) & \left.=1 G_{r}(x+z)-G_{r}(-x-z)+G_{r}(-z-x)-G_{r}(x-z)\right] \ell_{r} \\
& =2 B_{r}(x)\left[G_{r}(z)-G_{r}(-z)\right] \ell_{r} .
\end{aligned}
$$

From this relation and (3.5) it follows that (3.8) holds if there exists $x \notin K_{r}$ such that the matrix $B_{r}(x)$ is nonsingular. The latter condition is met if

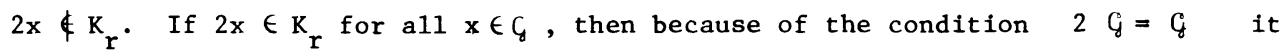
follows $x \in K_{r}$ for all $x$. Thus $\chi_{r}(x)=1$ for all $x$ contrary to our assumption.

Thus (3.8) is true for all $z \in C_{i}$ and Lemma 2 is proven.

Lemma 3. Assume that $x_{1}(x) \equiv 1$ and let $q=m_{1}$. Then $\ell_{1}(x)$ is a polynomial of degree $2 q-1$, which has the form

$$
\ell_{1}(x)=\sum_{k=0}^{q-1} \frac{M^{k}(x, x)}{(2 k+1) !} \psi_{1}(x)
$$

Here $M(x, y)$ are matrices of dimension $q$ under the following conditions:

$M\left(x_{1}+x_{2}, y\right)=M\left(x_{1}, y\right)+M\left(x_{2}, y\right), M(x, y)=M(y, x), Q_{1} M(x, x)=M^{t}(x, x) 0_{1} ;$

$M^{q}(x, x)=0, M^{2}(x, y)=M(x, x) M(y, y), M(x, y) \Psi_{1}(x)=M(x, x) \Psi_{1}(y)$ and

$\psi_{1}(\mathrm{x}+\mathrm{y})=\psi_{1}(\mathrm{x})+\psi_{1}(\mathrm{y}), \psi_{1} \in \mathfrak{J}^{\star} \mathrm{q}$.

Proof of Lemma 3. We have $B_{1}(x)=I+N(x)$, where $N^{q}(x)=0, q=m_{1}$.

Thus

$$
\ell_{1}(x+y)+\ell_{1}(x-y)-2 \ell_{1}(x)=2 N(y) \ell_{1}(x)
$$

and

$$
N(x+y)+N(x-y)-2 N(x)=2 N(y)+2 N(x) N(y) .
$$

The latter identity can be rewritten

$$
[L(y / 2)-L(-y / 2)]^{2} N(x)=2 N(y)[I+N(x)] .
$$

Easy induction shows that for $k=1,2, \ldots$

$$
[L(y / 2)-L(-y / 2)]^{2 k} \mathrm{~N}(\mathrm{x})=2^{k_{N} k}(y)[I+N(x)] \text {. }
$$

Thus in particular

$$
[L(y / 2)]-L(-y / 2)]^{2 q-2} N(x)=2^{q-1}{ }_{N}^{q-1}(y),
$$


which implies

$$
[I-L(y)]^{2 q-1} N(x)=0
$$

i.e., $N(x)$ is a polynomial of degree $2 q-2$. Because of the result mentioned in Section 2,

$$
N(x)=N_{2 q-2}(x)+\ldots+N_{2}(x),
$$

where for $k=1, \ldots, q-1$

$$
[\mathrm{L}(\mathrm{y} / 2)-\mathrm{L}(-\mathrm{y} / 2)]^{2 \mathrm{k}} \mathrm{N}_{2 \mathrm{k}}(\mathrm{x})=(2 \mathrm{k}) ! \mathrm{N}_{2 \mathrm{k}}(\mathrm{y}) \text {; }
$$

i.e., $\mathrm{N}_{2 \mathrm{k}}(\mathrm{x})$ is a homogenous polynomial of degree $2 \mathrm{k}, \mathrm{N}_{2 \mathrm{k}}(\mathrm{nx})=\mathrm{n}^{2 \mathrm{k}_{\mathrm{N}}}(\mathrm{x})$. These polynomials are defined by the formulas

$$
\mathrm{N}_{2 \mathrm{q}-2}(\mathrm{x})=\frac{1}{(2 \mathrm{q}-2) !}[\mathrm{L}(\mathrm{x} / 2)-\mathrm{L}(-\mathrm{x} / 2)]^{2 \mathrm{q}-2} \mathrm{~N}(\cdot),
$$

and for $k=q-2, \ldots, 1$

$$
\mathrm{N}_{2 \mathrm{k}}(\mathrm{x})=\frac{1}{(2 \mathrm{k}) !}[\mathrm{L}(\mathrm{x} / 2)-\mathrm{L}(-\mathrm{x} / 2)]^{2 \mathrm{k}}\left[\mathrm{N}(\cdot)-\mathrm{N}_{2 \mathrm{q}-2}(\cdot)-\ldots-\mathrm{N}_{2 \mathrm{k}+2}(\cdot)\right] \text {. }
$$

We prove at first that

$$
N_{2 k}(x)=\sum_{j=k}^{q-1} d_{j k}[2 N(x)]^{j} /(2 j) !
$$

where the coefficients $d_{j k}$ can be found in the following way. If $D$ is the lower triangular matrix formed by $d_{j k} k \leq j$, then $D=P^{-1}$ where the elements $P_{j k}$ of $P$ have the form

(Clearly $\mathrm{p}_{\mathrm{jk}}=0$ if $\left.\mathrm{k}>\mathrm{j}\right)$.

$$
p_{j k}=\frac{1}{(2 k) !} \sum_{i=0}^{2 k}\left(\begin{array}{c}
2 k \\
i
\end{array}\right)(-1)^{i}(i-k)^{2 j} \text {. }
$$

Indeed,

$$
\mathrm{N}_{2 q-2}(\mathrm{x})=\frac{1}{(2 q-2) !}[\mathrm{L}(\mathrm{x} / 2)-\mathrm{L}(-\mathrm{x} / 2)]^{2 \mathrm{q}-2} \mathrm{~N}(\cdot)=\frac{[2 \mathrm{~N}(\mathrm{x})]^{\mathrm{q}-1}}{(2 \mathrm{q}-2) !},
$$

so that $\mathrm{d}_{\mathrm{q}-1 \mathrm{q}-1}=1$.

Also,

$$
\begin{aligned}
{[L(x / 2)-L(-x / 2)]^{2 k_{N_{2 j}}(0)} } & =\sum_{i=0}^{2 k}\left(\begin{array}{c}
2 k \\
i
\end{array}\right)(-1)^{i} N_{2 j}((k-i) x) \\
& =\sum_{i=0}^{2 k}\left(\begin{array}{c}
2 k \\
i
\end{array}\right)(-1)^{i}(i-k)^{2 j} N_{2 j}(x) \\
& =(2 k) ! p_{j k} N_{2 j}(x) .
\end{aligned}
$$


Thus

$$
\begin{aligned}
N_{2 k}(x) & =\frac{1}{(2 k) !}[L(x / 2)-L(-x / 2)]^{2 k}\left[N(0)-N_{2 q-2}(0)-\ldots-N_{2 k+2}(0)\right] \\
& =[2 N(x)]^{k} /(2 k) !-\sum_{j=k+1}^{q-1} p_{j k} N_{2 j}(x)
\end{aligned}
$$

and it follows by induction that

$$
\begin{aligned}
& d_{j k}=-\sum_{i=k+1}^{j} d_{j i} p_{i k}, j>k \\
& d_{j j}=1 .
\end{aligned}
$$

But these identities mean $D=-D(P-I)+I$ or $D P=I$.

We prove now that

Indeed

$$
[L(y / 2)-L(-y / 2)]^{2} N_{2 k}(x)=2\left[N_{2 k}(y)+\sum_{j<k} N_{2 j}(y) N_{2(k-j)}(x)\right] .
$$

$$
\begin{aligned}
{[L(y / 2)-L(-y / 2)]^{2}[2 N(x)]^{k} \ell_{1}(z) } \\
=[L(y / 2)-L(-y / 2)]^{2} \sum_{i=0}^{2 k}\left(\begin{array}{c}
2 k \\
i
\end{array}\right)(-1)^{i} \ell_{1}(z+(i-k) x) \\
=\sum_{i=0}^{2 k}\left(\begin{array}{c}
2 k \\
i
\end{array}\right)(-1)^{i} 2 N((i-k) y) l_{1}(z+(i-k) x) \\
=\sum_{i=0}^{k-1}\left(\begin{array}{c}
i \\
i
\end{array}\right)(-1)^{i} 2 N((i-k) y)\left[l_{1}(z+(i-k) x)+\ell_{1}(z-(i-k) x)\right] \\
=\sum_{i=0}^{k-1}\left(\begin{array}{c}
2 k \\
i
\end{array}\right)(-1)^{i} 4 N((i-k) y) N((i-k) x) l_{1}(z) \\
\left.+\sum_{i=0}^{k-1} \begin{array}{c}
2 k \\
i
\end{array}\right)(-1)^{i} 2 N((i-k) y) l_{1}(z) \\
\left.=\sum_{i=0}^{2 k}\left(\begin{array}{c}
2 k \\
i
\end{array}\right)(-1)^{i} 2 N((i-k) y) N_{1}(i-k) x\right) l_{1}(z) \\
+\sum_{i=0}^{2 k}\left(\begin{array}{c}
2 k \\
i
\end{array}\right)(-1)^{i} 2 N((i-k) y) l_{1}(z) .
\end{aligned}
$$

Therefore

$[L(y / 2)-L(-y / 2)]^{2}[2 N(x)]^{k}=2(2 k) ! \sum_{j, i} p_{j+i k} N_{2 j}(y) N_{2 i}(x)+2(2 k) ! \sum_{j} p_{j k} N_{2 j}(y)$

and

$[L(y / 2)-L(-y / 2)]^{2} N_{2 k}(x)=\sum_{j=k}^{q-1} d_{j k}[L(y / 2)-L(-y / z)]^{2} \frac{[2 N(x)]^{j}}{(2 j) !}$ 


$$
\begin{aligned}
& =2 \sum_{j=k}^{q-1} d_{j k} \sum_{n, i} p_{n+i j} N_{2 N}(y) N_{2 i}(x)+2 \sum_{j=k}^{q-1} d_{j k} \sum_{i} p_{i j} N_{2 i}(y) \\
& =2\left[\sum_{i<k} N_{2 i}(y) N_{2(k-i)}(y)+N_{2 k}(y)\right] .
\end{aligned}
$$

Using (3.10) repeatedly we can now establish the following formula

$$
\begin{aligned}
{[L(y / 2)-L(-y / 2)]^{2 k_{N}}(\cdot) } & =2^{k} j_{1}+\ldots \sum_{7 j_{k}}=k N_{2 j_{1}}(y) \ldots N_{2 j_{k}}(y) \\
& =\left[2 N_{2}(y)\right]^{k},
\end{aligned}
$$

which gives the basic result:

$$
\begin{aligned}
& \mathrm{N}_{2 \mathrm{k}}(\mathrm{y})=\frac{1}{(2 \mathrm{k}) !}[\mathrm{L}(\mathrm{y} / 2)-\mathrm{L}(-\mathrm{y} / 2)]^{2 \mathrm{k}_{\mathrm{N}}} \mathrm{N}_{2 \mathrm{k}}(\cdot)=\frac{\left[2 \mathrm{~N}_{2}(\mathrm{y})\right]^{\mathrm{k}}}{(2 \mathrm{k}) !} .
\end{aligned}
$$

Note that there exists a function $M(x, y)$ on $\mathcal{C}_{d} \mathbb{C}_{\mathrm{g}}$ with values in $\mathfrak{F}_{\mathrm{q}}$ such that $2 \mathrm{~N}_{2}(\mathrm{x})=\mathrm{M}(\mathrm{x}, \mathrm{x})$ and it possesses the properties from the condition of Lemma 3.

Now we return to the equation (3.9) which can be rewritten in the following form

$$
[\mathrm{L}(\mathrm{y} / 2)-\mathrm{L}(-\mathrm{y} / 2)] \ell_{1}(\mathrm{x})=2 \mathrm{~N}(\mathrm{y}) \ell_{1}(\mathrm{x}) .
$$

It is easy to check that for $k=1,2, \ldots$

$$
[\mathrm{L}(\mathrm{y} / 2)-\mathrm{L}(-\mathrm{y} / 2)]^{2 \mathrm{k}} \ell_{1}(\mathrm{x})=[2 \mathrm{~N}(\mathrm{y})]^{\mathrm{k}} \ell_{1}(\mathrm{x}) .
$$

Thus

$$
[L(y / 2)-L(-y / 2)]^{2 q} \ell_{1}(x)=0,
$$

and $\ell_{1}(x)$ is a polynomial of degree $2 q-1$.

Analogously to previous considerations,

$$
e_{1}(x)=4_{2 q-1}(x)+\ldots+\psi_{1}(x),
$$

where $\varphi_{2 k+1}(x)$ is an homogenous polynomial of degree $2 k+1$, $\Psi_{2 k+1}(n x)=n^{2 k+1} \Psi_{2 k+1}(x)$.

Note, that if $2 x=2 y$, then $\psi_{2 k+1}(x)=\varphi_{2 k+1}(y), k=0,1, \ldots, q-1$. Thus the function $\ell(x)=2 \ell_{1}(x / 2)$ is defined.

Similarly to (3.10) we prove

$$
\Psi_{2 k+1}(x)=\sum_{j=k}^{q-1} c_{j k}[(2 j+1) !]^{-1}[2 N(x)]^{j} \ell(x),
$$

where the lower triangular matrix $C$ formed by the coefficients $c_{j k}, k \leq j$ has the form $C=V^{-1}$. Here $V$ is the matrix with elements 


$$
\begin{aligned}
& v_{j k}=\frac{2}{(2 k+1) !} \quad \sum_{1=0}^{2 k}\left(\begin{array}{c}
2 k \\
i
\end{array}\right)(-1)^{i}(i-k+1 / 2)^{2 j+1} . \\
& \text { Clearly } v_{j k}=0 \text { if } k>j \text {. } \\
& \text { Also } \\
& {[L(y / 2)-L(-y / 2)]^{2}[2 N(x)]^{j} \ell(x)} \\
& =2[L(y / 2)-L(-y / 2)]^{2} \sum_{i=0}^{2 j}\left(\begin{array}{c}
2 j \\
i
\end{array}\right)(-1)^{i} \ell_{1}((i-j) x+x / 2) \\
& =2 \sum_{i=0}^{2 j}\left(\begin{array}{c}
2 j \\
i
\end{array}\right)(-1)^{i} 2 N((i-j+1 / 2) y) \ell_{1}((i-j+1 / 2) x) \\
& =4 \sum_{i=0}^{2 j}\left(\begin{array}{c}
2 j \\
i
\end{array}\right)(-1)^{i} \sum_{n, k} N_{2 n}(y) \varphi_{2 k+1}(x)(i-j+1 / 2)^{2 n+2 k+1} \text {, }
\end{aligned}
$$

so that

$$
\begin{aligned}
{[L(y / 2)-L(-y / 2)]^{2} \varphi_{2 k+1}(x) } & =2 \sum_{j \leq k} c_{j k} v_{n+i j} \sum_{n, i} N_{2 n}(y) \varphi_{2 i+1}(x) \\
& =2 \Sigma N_{2(k-i)}(y) \varphi_{2 i+1}(x) .
\end{aligned}
$$

Using this identity repeatedly one obtains

$$
\begin{aligned}
{[L(y / 2)-L(-y / 2)]^{2 k} \varphi_{2 k+1}(x) } & =2^{k}{ }_{i_{1}}+\ldots+\sum_{k}+i_{k+1}=k{ }^{N} i_{1}(y) \ldots N_{2 i_{k}}(y) \varphi_{2 i_{k+1}+1}(x) \\
& =\left[2 N_{2}(y)\right]^{k} \varphi_{1}(x)
\end{aligned}
$$

and

$$
[\mathrm{L}(\mathrm{y} / 2)-\mathrm{L}(-\mathrm{y} / 2)]^{2 \mathrm{k}+1} \boldsymbol{\varphi}_{2 \mathrm{k}+1}(\mathrm{x})=\left[2 \mathrm{~N}_{2}(\mathrm{y})\right]^{\mathrm{k}} \Psi_{1}(\mathrm{y}) .
$$

Therefore

$$
\Psi_{2 k+1}(x)=\frac{\left[2 N_{2}(x)\right]^{k}}{(2 k+1) !} \varphi_{1}(x), \quad k=0,1, \ldots, q-1,
$$

and

$$
\ell_{1}(x)=\sum_{k=0}^{q-1} \frac{\left[2 N_{2}(x)\right]^{k}}{(2 k+1) !} \varphi_{1}(x) .
$$

The relation (3.13) for $k=1$ implies that

$$
M(x, y) \varphi_{1}(y)=M(y, y) \varphi_{1}(x) .
$$

Since $\varphi_{1}(x)$ is a polynomial of degree one, $\varphi_{1}$ is an additive homomorphism. Thus we proved Lemma 3.

Proof of Theorem 1. Now we are able to obtain the desired formula for the 
solution of (1.2). Clearly

$$
f(x)=f_{1}(x)+f_{2}(x),
$$

where $f_{1}(x)=[f(x)+f(-x)] / 2, f_{2}(x)=[f(x)-f(-x)] / 2$. It follows from $(1.2)$ that

$$
f_{1}(x+y)-f_{1}(x-y)=\langle[h(x)-h(-x)] / 2, k(y)\rangle=\langle w(x), k(y)\rangle
$$

and

$$
\mathrm{f}_{2}(\mathrm{x})=\langle\mathrm{h}(0), \mathrm{k}(\mathrm{x})\rangle=\langle\mathrm{h}, \mathrm{k}(\mathrm{x})\rangle \text {. }
$$

Thus $f_{1}(x)$ is a symmetric solution of the functional equation of Lemma 1 so that the vector functions $k(x)$ and $\ell(x)$ possess all properties given in the Lemmas. Therefore

$$
\begin{aligned}
& f(x)-f(0)=f_{1}(x)-f_{1}(0)+f_{2}(x) \\
& =\langle\mathrm{h}(\mathrm{x} / 2), \mathrm{k}(\mathrm{x} / 2)\rangle \\
& =\langle w(x / 2), k(x / 2)\rangle+\langle h, k(x)\rangle \\
& =\langle Q \ell(x / 2), \ell(x / 2)\rangle+\langle T h, \ell(x)\rangle \\
& =\sum_{r=1}^{R}\left[\left\langle Q_{r} \ell_{r}(x / 2), \ell_{r}(x / 2)>+<f_{r}, l_{r}(x)>\right]\right. \\
& =\left\langle\mathrm{f}_{1}, \sum_{\mathrm{k}=0}^{\mathrm{m}_{1}-1} \frac{{ }^{\mu} \mathrm{k}(\mathrm{x} / 2, \mathrm{x} / 2)}{(2 \mathrm{k}+1) !} \psi(\mathrm{x})\right\rangle \\
& +\frac{1}{4}<\sum_{k=0}^{m_{1}^{-1}} \frac{\left[M^{t}(x / 2, x / 2)\right]^{k}}{(2 k+1) !} Q_{1} \Psi(x), \sum_{i=0}^{m_{1}-1} \frac{M^{i}(x / 2, x / 2)}{(2 i+1) !} \Psi(x)> \\
& +\sum_{r=2}^{R}\left\{<\left[G_{r}^{t}(x / 2)-G_{r}^{t}(-x / 2)\right] Q_{r} e_{r},\left[G_{r}(x / 2)-G_{r}(-x / 2)\right] \ell_{r}\right\rangle \\
& \left.+<f_{r},\left[G_{r}(x)-G_{r}(-x)\right]>\right\} \\
& =\left\langle\sum_{k=0}^{m_{1}-1} \frac{\left[M^{t}(x, x)\right]^{k}}{(2 k+1) !} f_{1}, \psi(x)>+<\sum_{k=0}^{m_{1}-1} \frac{\left[M^{t}(x, x)\right]^{k}}{(2 k+2) !} Q_{1}(x), \varphi(x)>\right. \\
& \left.+\sum_{r=2}^{R} l<c_{r}, G_{r}(x) l_{r}>+<d_{r}, G_{r}(-x) l_{r}>+<c_{r}-d_{r}, l_{r}>\right] \text {. }
\end{aligned}
$$

We used here the formula 


$$
\left.\sum_{k=0}^{\mathrm{q}-1} \frac{M^{\mathrm{k}}(\mathrm{x} / 2, \mathrm{x} / 2)}{(2 \mathrm{k}+1) !}\right]^{2}=2 \sum_{\mathrm{k}=0}^{\mathrm{q}-1} \frac{\mathrm{M}^{\mathrm{k}}(\mathrm{x}, \mathrm{x})}{(2 \mathrm{k}+2) !},
$$

which follows from the properties of $M(x, x)$.

The formula (3.1) follows with $H(x, x)=M^{t}(x, x)$ and $F_{r}(x)=G_{r}^{t}(x)$.

We prove now that every function $f$ of the form (3.1) satisfies the equation

(1.3). Note that for $k \geq 1$

$$
\begin{aligned}
& H^{k}(x+y, x+y)-H^{k}(x-y, x-y) \\
& =2 \underset{i: k-i}{ } \operatorname{odd}\left(\begin{array}{l}
k \\
i
\end{array}\right)[H(x, x)+H(y, y)]^{i}[2 H(x, y)]^{k-i} \\
& =2 \sum_{i: k-i} \text { odd, } j \leq i\left(\begin{array}{l}
k \\
i
\end{array}\right)\left(\begin{array}{l}
i \\
j
\end{array}\right)[H(x, x)]^{j+(k-i-1) / 2}[H(y, y)]^{i-j+(k-i-1) / 2} 2^{k-i} H(x, y) \\
& =2 \sum_{i+1 \leq k}\left(\begin{array}{c}
2 k \\
2 i+1
\end{array}\right)[H(x, x)]^{i}[H(y, y)]^{k-1-i} H(x, y) \text {. }
\end{aligned}
$$

The last identity follows from the formula

$$
\sum_{\substack{i: k-i \text { odd } \\
2 j+k-i=2 p-1}}\left(\begin{array}{l}
k \\
i
\end{array}\right)\left(\begin{array}{l}
i \\
j
\end{array}\right) 2^{k-i}=\left(\begin{array}{c}
2 k \\
2 p-1
\end{array}\right),
$$

which is easily obtained by comparison of coefficients of $a^{2 p_{b}}{ }^{2 k-2 p}$ in expansions $\left(a^{2}+b^{2}+2 a b\right)^{k}-\left(a^{2}+b^{2}-2 a b\right)^{k}$ and $(a+b)^{2 k}-(a-b)^{2 k}$.

Also,

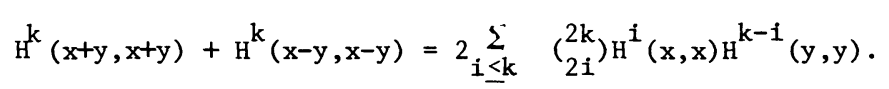

Using this formula, (3.14) and properties of the function $\mathrm{H}$ one obtains

$$
\begin{aligned}
f(x+y)-f(x-y) & =2<\sum_{k=0}^{m_{1}-1} \frac{H^{k}(x, x)}{(2 k) !} f_{1}+\sum_{k=0}^{m_{1}^{-1}} \frac{H^{k}(x, x)}{(2 k+1) !} Q_{1} \psi(x), \sum_{i=0}^{m_{1}^{-1}} \frac{\left[H^{t}(y, y)\right]^{i}}{(2 i+1) !} \psi(y)> \\
& +\sum_{r=2}^{R}<F_{r}(x) c_{r}-F_{r}(-x) d_{r},\left[F_{r}^{t}(y)-F_{r}^{t}(-y)\right] e_{r}>
\end{aligned}
$$

Thus (1.2) holds and the statements of the Theorem 1 about vectors $\mathrm{s}^{\mathrm{t}}(\mathrm{x}) \psi(\mathrm{x})$, $C(x) f_{1}+S(x) Q_{1} \varphi(x), F_{r}(x) c_{r}-F_{r}(-x) d_{r}$ and $\left[F_{r}^{t}(x)-F_{r}^{t}(-x)\right] \ell_{r}, x \in c_{i}, r=2, \ldots, R$ follow from the assumed linear independence of functions $h_{j}, k_{j}, j=1, \ldots, m$. The uniqueness up to equivalence of the matrices in formula (3.1) is a corollary of the uniqueness of the decomposition of the space $\mathcal{J}^{\mathrm{m}}$ into direct sum of subspaces invariant with 
respect to commuting matrices $A(x)$ from (3.4). Theorem 1 is proved.

REMARK 1. If $\mathrm{f}$ is a topological group and $\mathrm{f}$ (or $\mathrm{g}$ ) is assumed to be a continuous (or only a measurable) function, then the condition $2 C_{d}=y$ of the Theorem 1 (or 2) can be replaced by the following one: the subgroup $2 \zeta$ is dense in $C$. Incidentally, this condition means that the dual group does not have elements of order two.

REMARK 2. Theorems 1 and 2 are true if the field $\mathfrak{f}$ is not algebraically closed. In this case all homomorphisms from $\zeta$ into corresponding vector spaces over $\mathfrak{F}$ should be replaced by homomorphisms from $\zeta_{\delta}$ into vector spaces over a finite extension of the field $\mathfrak{F}$. Of course if $\mathfrak{F}$ is the field of reals, this extension coincides with the field of complex numbers.

For instance, any solution of the classical D'Alembert's equation (1.4) has the form $[\chi(x)+\chi(-x)] / 2$ where $\chi$ is a multiplicative homomorphism into a simple extension of the initial field $\mathfrak{F}$.

REMARK 3. The general form of a solution of (1.1) easily follows from Theorems 1 and 2. Namely, if $\mathrm{m}$ and $\mathrm{p}$ denote the maximal number of linearly independent functions among $\alpha_{j}(x), \beta_{j}(y)-\beta_{j}(-y)$ and among $\alpha_{j}(x), \beta_{j}(y)+\beta_{j}(-y), j=1, \ldots, n$, respectively, then

$$
\phi_{1}(x)=[f(x)+g(x)] / 2, \phi_{2}(x)=[f(x)-g(x)] / 2
$$

where the forms of $f(x)$ and $g(x)$ are given in Theorems 1 and 2 .

\section{DISCUSSION.}

It follows from the proof of Theorem 1 that every solution $f$ of (1.2) has the form

$$
f(x)=\langle L(x) f, \Delta\rangle \text {. }
$$

Here $L$ is a cyclic representation of the group $C$ in the space $V$ with a cyclic vector $f$, and the space $V_{-}$spanned by the vectors $[L(x)-L(-x)] f, x \in C$ has dimension m. The element $\Delta$ of the dual space $V^{\star}$ is a cyclic vector for the contragradient representation $L^{*}, L^{*}(x)=L^{t}(-x)$. (Indeed we define $\Delta$ in the following way: $\left\langle\mathrm{h}, \Delta>=\mathrm{h}(0)\right.$ for all $\mathrm{h}$ from $\mathrm{V}$. Then $\left\langle\mathrm{h}, \mathrm{L}^{*}(\mathrm{x}) \Delta\right\rangle=\mathrm{h}(-\mathrm{x})$ and the vectors $L^{*}(x) \Delta, x \in G$ must span the whole space $v^{*}$.) Clearly the representation $L$ under these conditions is defined uniquely up to equivalence. A natural question 
is whether the representation $\mathrm{L}$ is finite dimensional. Bounds for the dimension of $\mathrm{L}$ in terms of $\mathrm{m}$ are also of interest. The same question can be formulated for the functional equation (1.3)

It was proved in [13] that for both equations the space $V$ is finite dimensional if $G$ is a compact group. In the non-locally compact case the situation for the equations (1.2) and (1.3) is different. Here is an example of a solution to (1.3) with infinite dimensional representation $\mathrm{L}$.

Let $\mathcal{G}$ be an infinite dimensional Hilbert space, $g(x)=\|x\|^{2}$. Then

$$
g(x+y)+g(x-y)=2\left(|| x\left\|^{2}+\right\| y \|^{2}\right),
$$

so that (1.3) holds, and the dimension of the subspace $v_{+}$spanned by the vectors $[L(x)+L(-x)] g, x \in \mathcal{G}$ is two, and $g(x)$ is a polynomial of degree two.

However

$$
g(x+y)-g(x-y)=4<x, y>\text {, }
$$

and the space $V_{-}$is an infinite dimensional one. Therefore $V=V(g)$ is an infinite dimensional space as well. Thus not every polynomial solves (1.2) or (1.5).

Note that in this example the homomorphism $\psi$ of Theorem 2 is zero. Also note that if $g$ is an odd function, $g(-x)=-g(x)$, and $g$ satisfies (1.3), then

$$
g(x+y)-g(x-y)=g(x+y)+g(y-x)=\langle u(y), v(x)\rangle,
$$

so that $g$ also satisfies (1.2). Thus both spaces $v_{+}$and $v_{-}$have dimension $p$, and the dimension of $\mathrm{V}$ does not exceed $2 \mathrm{p}$. Of course the same remark refers to equation (1.2).

Now let $f$ be a solution of (1.2). Then $F$ has the form (3.1), and

$$
\begin{aligned}
F(x+y)+f(x-y) & =2<C(y) f_{1}, S^{t}(x) \phi(x)> \\
& +2<H(x, y) T(x) Q_{1} \varphi(x), T^{t}(y) \varphi(y)>+2<T(x) Q_{1} \varphi(x), \varphi(y)> \\
& +2<T(y) Q_{1} \varphi(y), \varphi(y)>+\sum_{r=2}^{R}<F_{r}(x) f_{r}+F_{r}(-x) d_{r},\left[F_{r}^{t}(x)+F_{r}^{t}(-x)\right] \ell_{r}>.
\end{aligned}
$$

The proof of (4.2) is analogous to that of the identity (3.15).

Note that the second term in (4.1) has the form

$$
\begin{aligned}
\left\langle H(x, y) T(x) Q_{1} \varphi(x), T^{t}(y) \varphi(y)>\right. & =\left\langle H(x, x) T(x) Q_{1} \Psi(y), T^{t}(y) \Psi(y)\right\rangle \\
& =\sum_{i, j=1}^{1} \alpha_{i j}(x) \Psi_{i}(y) \eta_{j}(y),
\end{aligned}
$$


where $\alpha_{i j}(x)$ are elements of the matrix $H(x, x) T(x) Q_{1}$ and $\psi_{i}(y)$ and $\eta_{j}(y)$ are coordinates of the functions $\psi(y)$ and $T^{t}(y) \varphi(y)$.

Therefore the dimension of the space $v_{+}$does not exceed

Thus

$$
\mathrm{m}_{1}+\mathrm{m}_{1}^{2}+2+\sum_{\mathrm{r}=2}^{\mathrm{R}} \mathrm{m}_{\mathrm{r}}=\mathrm{m}_{1}^{2}+\mathrm{m}+2 \text {. }
$$

$$
\operatorname{dim} V(f) \leq m_{1}^{2}+2 m+2 \leq m^{2}+2 m+2
$$

and the next result follows.

Theorem 3. Every solution $f$ of the equation (1.2) has the form (4.1) with a finite dimensional representation $L, \operatorname{dim} L \leq \mathrm{m}^{2}+\mathrm{m}+2$. The representation $\mathrm{L}$ is defined uniquely up to equivalence.

Theorem 4. Every solution $g$ of the equation (1.3) has the form (4.1) with a finite dimensional representation $\mathrm{L}$ under one of the two following conditions:

(i) $g(-x)=-g(x), \quad x \in \mathcal{C}$,

(ii) $\operatorname{dim} \operatorname{Hom}\left(\mathcal{G}, \mathfrak{F}^{\mathrm{n}}\right)=\rho_{\mathrm{n}}<\infty$ for $\mathrm{n}=1,2, \ldots$.

Under condition (i) dim $L \leq 2 \mathrm{p}$; under condition (ii) $\operatorname{dim} \mathrm{L} \leq \mathrm{p}\left(\rho_{\mathrm{p}}+2\right)$.

The proof of Theorem 4 under condition (ii) follows from the following formula valid for any solution of (1.3)

$$
\begin{aligned}
g(x+y)-g(x-y) & =2<H(x, y) S(x) Q_{1} a_{1}, S^{t}(y) a_{1}>+2<S(y) \psi(y), C^{t}(x) a_{1}> \\
& \left.+\sum_{r=2}^{R}<L F_{r}(x) g_{r}-F_{r}(-x) b_{r},\left[F_{r}^{t}(y)-F_{r}^{t}(-y)\right] a_{r}\right\rangle .
\end{aligned}
$$

This identity implies, that the dimension of the subspace $V_{-}$is less or equal to $\mathrm{p}_{1} \rho_{\mathrm{p}_{1}}+\mathrm{p}_{1}+\sum_{\mathrm{r}=2}^{\mathrm{R}} \mathrm{p}_{\mathrm{r}}=\mathrm{p}+\mathrm{p}_{1} \rho_{\mathrm{p}_{1}}$.

Therefore

$$
\operatorname{dim} V(g) \leq p+p_{1}\left(\rho_{p_{1}}+1\right) \leq p\left(\rho_{p}+2\right)
$$

and Theorem 2 follows.

Assume now that $\zeta$ is a topological group and continuous solutions of equations (1.2) and (1.3) are considered. The $\varphi(x)=0$ for all $x$ belonging to a compact subgroup of $C_{f}$. Therefore the first term in the formula (3.1) vanishes if $C$ is a compact group.

If the group $\zeta$ does not contain nontrivial compact groups, then any matrix homomorphism $F(x)$ has the form $F(x)=\exp \{H(x)\}$ where $H \in \operatorname{Hom}\left(\zeta_{y}, \mathcal{F}_{h}\right)$. (cf. [5p. 
393] for one dimensional result.) In this case, the power series for, say, $[F(x)+F(-x)] / 2$ bears some resemblance to the function $C(x)$ and explains the structure of the latter.

Thus if $G$ is a compact commutative group, it follows from Theorem 1 , that every solution of (1.2) has the form

$$
f(x)=\sum_{k=1}\left[c_{k} x_{k}(x)+d_{k} x_{k}(-x)\right]
$$

where $x_{k}(x+y)=x_{k}(x) x_{k}(y), k=1, \ldots, m$ are different multiplicative homomorphisms of $c$. The same is true for equation (1.3).

As another application of Theorems 1 and 2 notice that every solution of (1.2) or (1.3) is an exponential polynomial. (However, as we noticed, not every exponential polynomial can be a solution.) Indeed, the proof of Theorem 1 shows that $<S(x) f_{1}, \psi(x)>$ and $\left\langle T(x) Q_{1} \psi(x), \psi(x)>\right.$ are polynomials in components of $\psi(x)$ of degree $2 m$ and $F_{r}(x)=g_{r}(x)\left(I+C_{r}(x)\right)$ where $g_{r}(x)$ is a multiplicative homomorphism, I is the identity matrix, and the matrix $c_{r}(*)$ is a nilpotent one, $C_{r}^{m_{r}}(x)=0$. Thus

$$
\left\langle\mathrm{F}_{\mathrm{r}}(\mathrm{x}) \mathrm{f}_{\mathrm{r}},{ }_{\mathrm{r}}>=C_{\mathrm{r}}(\mathrm{x})<\mathrm{I}+\mathrm{C}_{\mathrm{r}}(\mathrm{x}) \mathrm{f}_{\mathrm{r}}, \mathrm{l}_{\mathrm{r}}>=\mathrm{g}_{\mathrm{r}}(\mathrm{x}) \mathrm{p}_{\mathrm{r}}(\mathrm{x})\right. \text {, }
$$

where $\mathrm{p}_{\mathrm{r}}(\mathrm{x})$ is a polynomial of degree $\mathrm{m}_{\mathrm{r}}$. In the case $C_{\mathrm{d}}=\mathrm{R}^{\mathrm{m}}$ one can indicate conditions on the coefficients of these polynomials (See [16]•).

ACKNOWLEDGEMENT. The author is grateful to Professor R.C. Penney for his interest in this work. This work was supported in part by National Science Foundation grant MCS 77 - 19640 and in part by National Science Foundation grant MCS - 7802300 .

\section{REFERENCES}

1. ACZEL, J. Lectures on functional equations and their applications, Academic Press, New York (1966).

2. ACZEL, J. Functions of Binomial Type Mapping Group ids into Rings, Math. Zeitschrift 154 (1977), pp. 115-124.

3. COROVEI, I. The cosine functional equation for nilpotent groups, Aequationes Math. 15 (1977), pp. 99-106.

4. ENGERT, M. Finite dimensional translation invariant subspaces, Pacific J. Math. 32 (1970), pp. 333-343.

5. HEWITT, E. and ROSS, K.A. Abstract Harmonic Analysis, Vo1. 1, Berlin-GotingenHeidelberg, Springer-Verlag (1963).

6. HOSSZU, M. Some remarks on the cosine functional equation, Pub1. Math. Debrecen 16 (1969), pp. 93-98. 
7. KANNAPPAN, P1. The functional equation $f(x y)+f\left(x y^{-1}\right)=2 f(x) f(y)$ for groups, Proc. Amer. Math. Soc. 19 (1968), pp. 69-74.

8. LAIRD, P.G. On characterizations of exponential polynomials, Pacific J. Math. 80 (1979) pp. 503-507.

9. LIJN, G. van der. La definition fonctionelle des polynomes dans les groupes abeliens, Fund. Math. 33 (1945), pp. 42-50.

10. McKIERNAN, M.A. "Equations of the form $H(x \circ y)=f_{i}(x) g_{i}(y)$," Aequationes Math. 16 (1977), pp. 51-58.

11. MCKIERNAN, M.A. "The Matrix Equation $a(x \circ y)=a(x)+a(x) a(y)+a(y), "$ Aequationes Math. 15 (1977), pp. 213-223.

12. O'CONNOR, T.A. A solution of D'Alembert's functional equation on a locally compact Abelian group, Aequationes Math. 15 (1977), pp. 235-238.

13. PENNY, R.C. and RUKHIN, A.L. D'Alembert's functional equation on groups, Proc. Amer. Math. Soc. 77 (1979), pp. 73-80.

14. REJTO, L. B-algebra valued solution of the cosine equation, Studia Sci. Math. Hung. 7 (1972), pp. 331-336.

15. RUKHIN, A.L. The families with a universal Bayesian estimator of the transformation parameter, Symposia Mathematica, Instituto Nationale di Alta Mathematica, Vo1. 21 (1977), pp. 19-26.

16. RUKHIN, A.L. Universal Estimators of a Vector Parameter, submitted to Journal of Multivariate Analysis.

17. STONE, J.J. Exponential polynomials on commutative semigroups, Appl. Math. and Stat. Lab. Technical Note, No. 14, Stanford University 1960.

18. SUPRUNENKO, D.A. and TYSHKEVICH, R.I. Commutative Matrices, Academic Press, New York, London, 1968. 


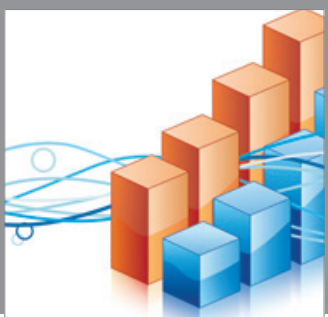

Advances in

Operations Research

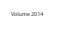

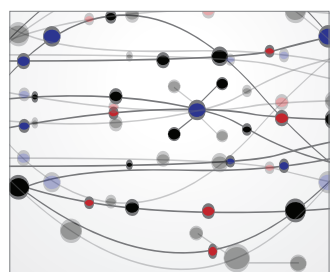

\section{The Scientific} World Journal
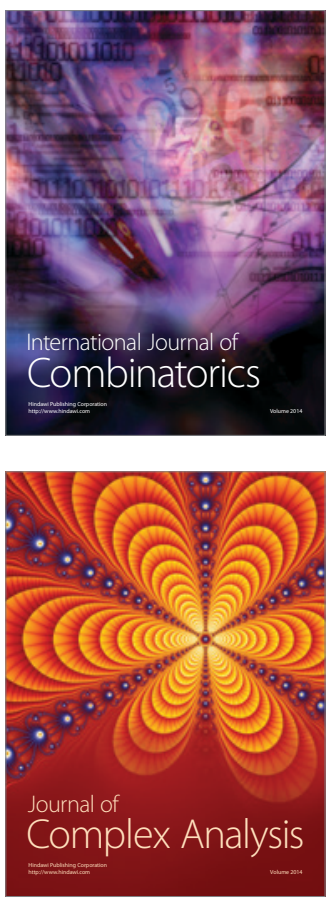

International Journal of

Mathematics and

Mathematical

Sciences
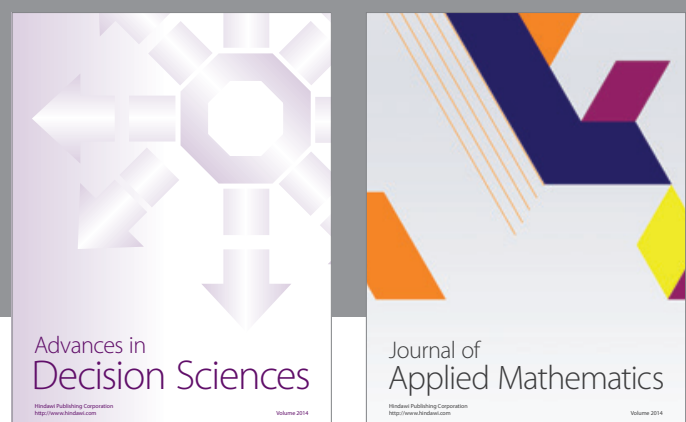

Journal of

Applied Mathematics
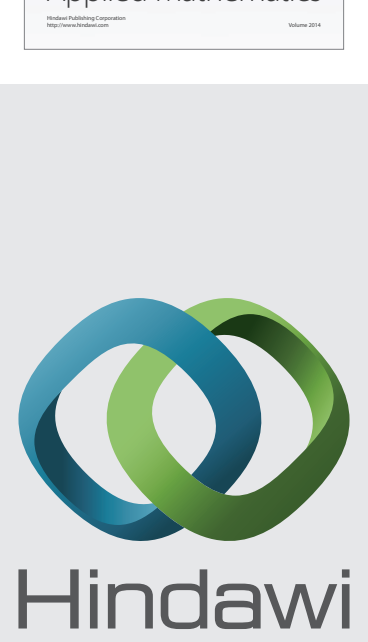

Submit your manuscripts at http://www.hindawi.com
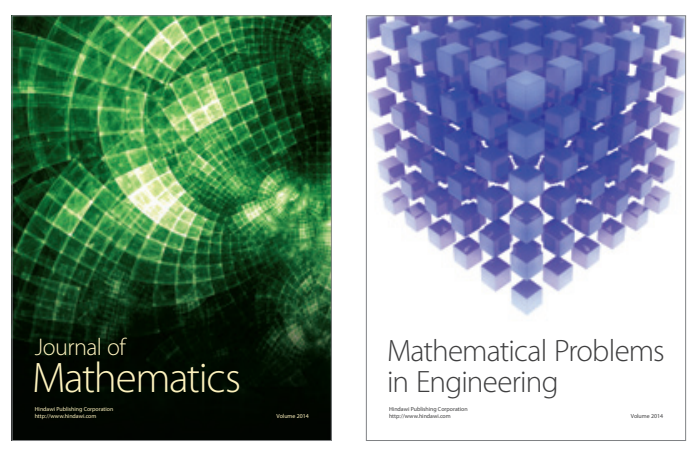

Mathematical Problems in Engineering
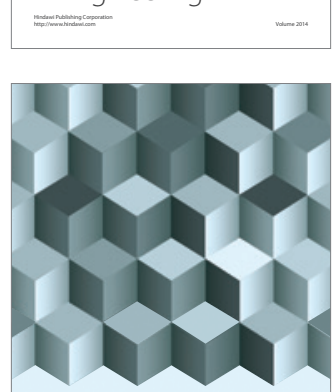

Journal of

Function Spaces
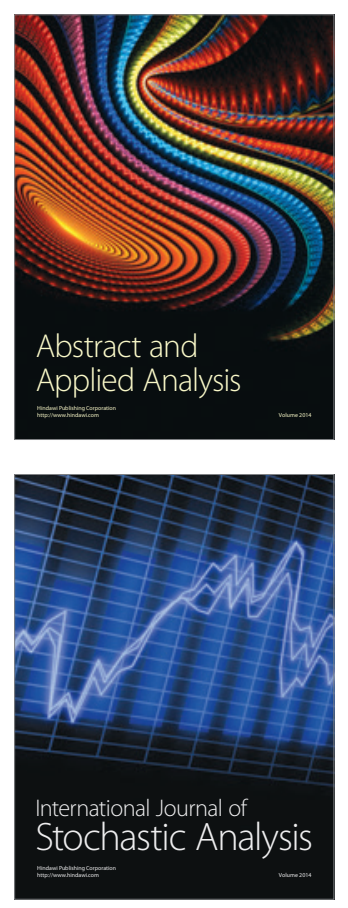

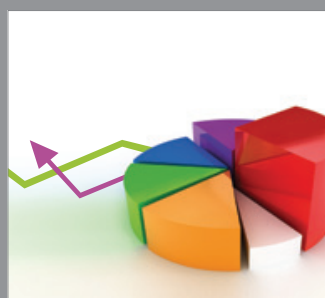

ournal of

Probability and Statistics

Promensencen
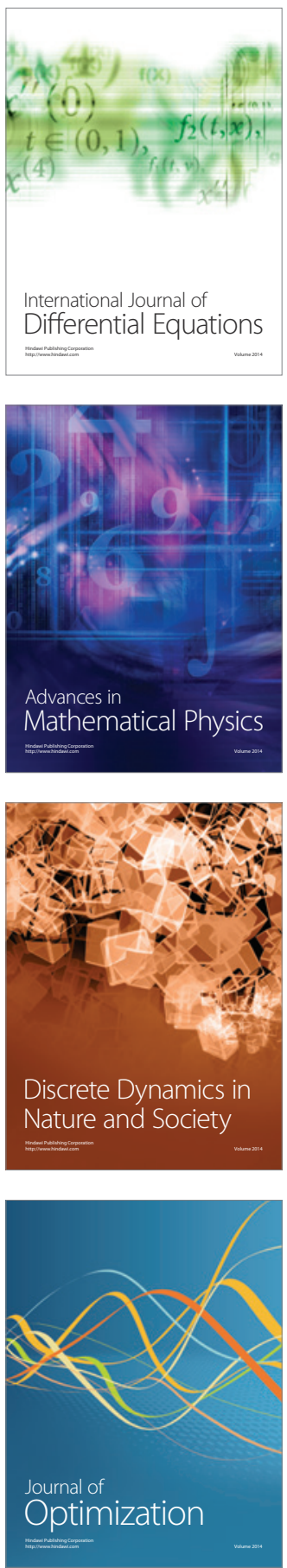